\title{
Ermittlung des Energiebedarfs zur Bewegung von Fahrzeugen in mikroskopischen Verkehrssimulationen
}

\author{
Peter Bernhardt* , Christian Sauer, Michael Hendrix
}

\section{Zusammenfassung}

Die Integration von Modellen für Fahrzeuge mit alternativen Antrieben in Verkehrssimulationen erfordert eine genauere Betrachtung der Energieflüsse in den einzelnen Fahrzeugen. Diese Arbeit betrachtet den Energiebedarf für die Bewegung von Fahrzeugen und evaluiert vorhandene klassische Modelle zur Abstraktion der physikalischen Einflüsse. Aufgrund der fehlenden Einstimmigkeit der Autoren bei der Beschreibung solcher Modelle in der Literatur wird letztlich der Ansatz verfolgt, ein entsprechendes Modell von der physikalischen Basis ausgehend neu zu entwickeln. Zusätzlich dazu wird festgestellt, dass die Beschränkungen der geläufigen Verkehrssimulationsumgebungen einen signifikanten Einfluss auf die Berechenbarkeit einzelner Komponenten derartiger Modelle haben. Das geschaffene Modell wird anschließend in verschiedenen Varianten in einem Vergleich mit einem weit verbreiteten Modell evaluiert. Zu guter Letzt muss konstatiert werden, dass eine Erhöhung der Realitätsnähe der Simulation - insbesondere im situativen Bereich - erreicht werden konnte, für wesentliche Verbesserungen jedoch eine Beseitigung bestehender Restriktionen der Simulationsumgebungen erforderlich wäre.

\section{Abstract}

The integration of models for alternative fuel vehicles in traffic simulation requires a closer examination of the energy flows in the individual vehicles. This work considers the energy required for the movement of vehicles and evaluates existing classical models for the abstraction of the physical influences. Due to the lack of unanimity of the authors in the discribing of such models in the literature, the approach ultimately pursued requires redeveloping an appropriate model of the physical basis as a starting point. Additionally, it has been established that the restrictions of the common traffic simulation environments have a significant impact on the computability of individual components of such models. The created model is then evaluated in different variants in comparison with a widely used model. Finally, it is notable that increasing simulation realism could be achieved, however, for in order to see substantial improvements, the elimination of certain restrictions of current simulation environments would be required.

\section{EINLEITUNG}

Das breite Bewusstsein für ökologische Thematiken auf der Welt und die dadurch entstehenden gesellschaftlichen Rahmenbedingungen haben in den letzten Jahren zu einer Verstärkung der Weiterentwicklung von Antriebskonzepten für Straßenfahrzeuge geführt. So werden den Käufern von Fahrzeugen mit klassischem Antriebskonzept, wie dem Verbrennungsmotor, in immer stärkerem Maße zusätzliche Angebote mit alternativen Antriebskonzepten, wie z.B.
Elektromotor oder Hybridantrieb, gemacht. Insbesondere Fortschritte bei den Technologien zur Speicherung elektrischer Energie, gerade hinsichtlich der Faktoren Kapazität und Kosten, erlauben es den Herstellern, die steigende Nachfrage nach alternativen Antriebsvarianten nicht nur exemplarisch, sondern in Serie zu bedienen. Auch wenn aktuell noch nicht von einer weiten Verbreitung ${ }^{1}$ von Fahrzeugen mit alternativem Antrieb gesprochen werden kann, so ist doch das ehrgeizige Ziel der Bundesregierung, bis zum Jahr 2020 eine Million Zulassungen von Elektrofahrzeugen in Deutschland zu erreichen (Die Bundesregierung 2009: 2). Unabhängig davon, dass der dann eventuell erreichte Anteil von knapp 2,0 Prozent am Gesamtfahrzeugbestand noch nicht signifikant erscheint, so kann und muss von einer punktuell - wie z. B. in Städten - und damit verkehrstechnisch relevanten Verbreitung von Fahrzeugen mit alternativen Antrieben ausgegangen werden. Die in Verkehrssimulationen eingesetzten Simulationsmodelle für Fahrzeuge müssen auf diese neuar-

\footnotetext{
${ }^{1}$ Im Jahr 2013 besaßen 1,5 Prozent der in Deutschland neu zugelassenen Fahrzeuge einen alternativen Antrieb (Kraftfahrt-Bundesamt 2014b). Wodurch am 01.01.2014 ein Gesamtanteil von 0,2 Prozent am Fahrzeugbestand erreicht wurde (Kraftfahrt-Bundesamt 2014a).
} 
tigen Gegebenheiten abgestimmt werden, damit sich auch in diesen Szenarien weiterhin aussagekräftige Ergebnisse erzielen lassen.

Dies bedeutet im Detail eine Abkehr von der Verwendung von statischen Modellen zur Simulation von Fahrzeugen mit klassischen Antriebskonzepten wie dem Verbrennungsmotor. Die Notwendigkeit dafür basiert auf der Feststellung, dass für die Simulation von Fahrzeugen mit alternativen Antriebskonzepten, wie z.B. Elektromotor oder Hybridantrieb, ein dynamisches Simulationsmodell benötigt wird. Dynamisch vor allem in der Hinsicht, dass bestimmte spezifische Eigenschaften eines Fahrzeuges nicht mehr konstant sind, sondern sich während der Fahrt verändern können. So hat zum Beispiel ein Hybridfahrzeug nicht mehr nur eine maximale Beschleunigung, sondern diese hängt in starkem Maße davon ab, welche Kombination von Antrieben situativ zum Einsatz kommt. Beispielhaft dafür steht der Toyota Prius III. Dessen Gesamtsystemleistung des Hybrid-Antriebs von $100 \mathrm{~kW}$ erlaubt andere Fahrleistungen als die reinen $73 \mathrm{~kW}$ an Maximalleistung des Verbrennungsmotors (Toyota Deutschland $\mathrm{GmbH}$ 2014: 18).

Ein Zwischenziel auf dem Weg zur Entwicklung eines solchen dynamischen Simulationsmodells für Fahrzeuge mit flexiblen Antriebskonzepten ist die Erarbeitung eines allgemeingültigen Modells zur Bestimmung des Energiebedarfs sich bewegender Fahrzeuge unter Berücksichtigung der Fähigkeiten von mikroskopischen Verkehrssimulationen. Ein besonderer Schwerpunkt liegt dabei auf der Ausgestaltung des Dilemmas zwischen physikalischer Genauigkeit und notwendiger rechentechnischer Performanz.

Dieser Beitrag gliedert sich wie folgt: Sektion II gibt einen Überblick über vorhandene Arbeiten zu diesem Thema und bewertet deren Ansätze. In Sektion III wird ein eigener Ansatz zur Ermittlung des Energiebedarfs hergeleitet, welcher darauf folgend in Sektion IV mit den bestehenden Arbeiten verglichen wird. Sektion V fasst dann die erzielten Ergebnisse zusammen und zeigt den weiteren Forschungsbedarf auf.

\section{STAND DER FORSCHUNG}

Die Ermittlung des Energiebedarfs von Kraftfahrzeugen spielt insbesondere bei der Betrachtung der ausgestoßenen Emissionen eine wichtige Rolle. Da dies keine neue Forschungsthematik ist, existieren verschiedene Ansätze der Modellierung. Treiber et. al. geben eine kompakte Übersicht über die möglichen Modellierungsformen (Treiber \& Kesting 2013: 379-387). Da in dieser Arbeit und der zugrunde liegenden Zielstellung eine Tank-to-Wheel-Betrachtung für einzelne Fahrzeuge verfolgt wird, kommen nur lastbasierte Modelle infrage. Dabei kann jedoch schon im Voraus festgelegt werden, dass das gesuchte physikbasierte Modell zwar mit etablierten Modellen, wie dem „Comprehensive Modal Emission Model“ (CMEM) oder dem „Passenger Car and Heavy Duty Emission Model“ (PHEM), vergleichbar ist, jedoch insbesondere aufgrund der Anforderung an die rechentechnische Performanz ohne deren tiefgreifenden Detailgrad auskommen muss.

Beispielhaft für eine Vielzahl von Veröffentlichungen steht das von Treiber \& Kesting (2013) beschriebene Treibstoffverbrauchsmodell, welches auf einem klassischen physikalischen Kräftemodell basiert. Dazu wird pro Zeitintervall ermittelt, wie hoch die Leistung ist, die das Fahrzeug im nächsten Schritt erbringen muss, um daraus dann den entsprechenden Treibstoffbedarf zu berechnen. Die physikalischen Einflüsse auf das Fahrzeug ergeben sich bei Treiber \& Kesting additiv aus Beschleunigungskraft, Hangabtriebskraft, Rollwiderstand und Luftwiderstand. Die Auswirkungen der Wirkungsgrade werden, mit Ausnahme beim Motor bei der Ermittlung des Treibstoffbedarfs, außer Acht gelassen.

Neudorfer (2010) setzt ebenfalls auf das physikalische Kräftemodell; erweitert dieses jedoch im Bereich des Rollwiderstandes um den Lagerwiderstand.

Im Rahmen der Simulation von Elektrofahrzeugen beschäftigen sich auch Maia et al. (2011) mit dem Energiebedarf von Fahrzeugen. Das physikalische Kräftemodell wird von ihnen insbesondere um die Berücksichti- gung der Kraft zur rotatorischen Beschleunigung des Motors erweitert. Zusätzlich zu den einwirkenden Kräften werden auch die Wirkungsgrade von Motor und Getriebe berücksichtigt. Bei der letztendlichen Berechnung des Energiebedarfs beschreibt diese Arbeit als einzige auch einen Regelkreis mit der Aufgabe, zu kontrollieren, ob der Motor überhaupt in der Lage ist, die angeforderte Leistung zu liefern und bei Bedarf die Anforderung entsprechend zu korrigieren.

In ihrer Untersuchung zu Ladeinfrastrukturen für elektrisch angetriebene Busse ermitteln Kurczveil \& Schnieder (2013) ebenfalls den notwendigen Energiebedarf. Wenngleich in dieser Arbeit nicht mit den Kräften, sondern den äquivalenten Energiemengen gearbeitet wird, so erweitern die Autoren das klassische Kräftemodell um den Widerstand zur Bewältigung von Kurven im Straßenverlauf. Obzwar der Einfluss des Kurvenwiderstands bei schweren Fahrzeugen wie Bussen sicherlich größer als bei Pkw ist, fällt es schwer, diesen von vornherein - wie in vielen anderen Arbeiten - von der Hand zu weisen. Vor allem da auch Hirano et al. (2014) die Auswirkungen des Kurvenwiderstands eindrucksvoll aufzeigen (vgl. Abbildung 2).

Beispielhaft für eine Berücksichtigung der Anforderungen an die Performanz steht die Arbeit von Schellenberg et al. (2014). Das dort entwickelte Modell zur Ermittlung des Energiebedarfs ist rein analytischer Natur und verwendet z. B. stets eine konstante Motoreffizienz für alle Fahrsituationen. Ebenso geht das physikalische Kräftemodell über den Standard nicht hinaus, was jedoch genau der Zielstellung der Arbeit entspricht.

Insgesamt gesehen ist keines dieser Modelle auf Fahrzeuge mit kombinierten Antrieben, wie z. B. Hybridfahrzeugen ausgerichtet. Dies bedeutet, dass immer von konstanten Eigenschaftswerten ausgegangen wird und Veränderungen der Kraftflüsse unberücksichtigt bleiben.

Im Ergebnis führt das Literaturstudium zu keinem einhelligen Gesamtbild. Obgleich die Mehrzahl aller Publikationen auf das klassische phy- 


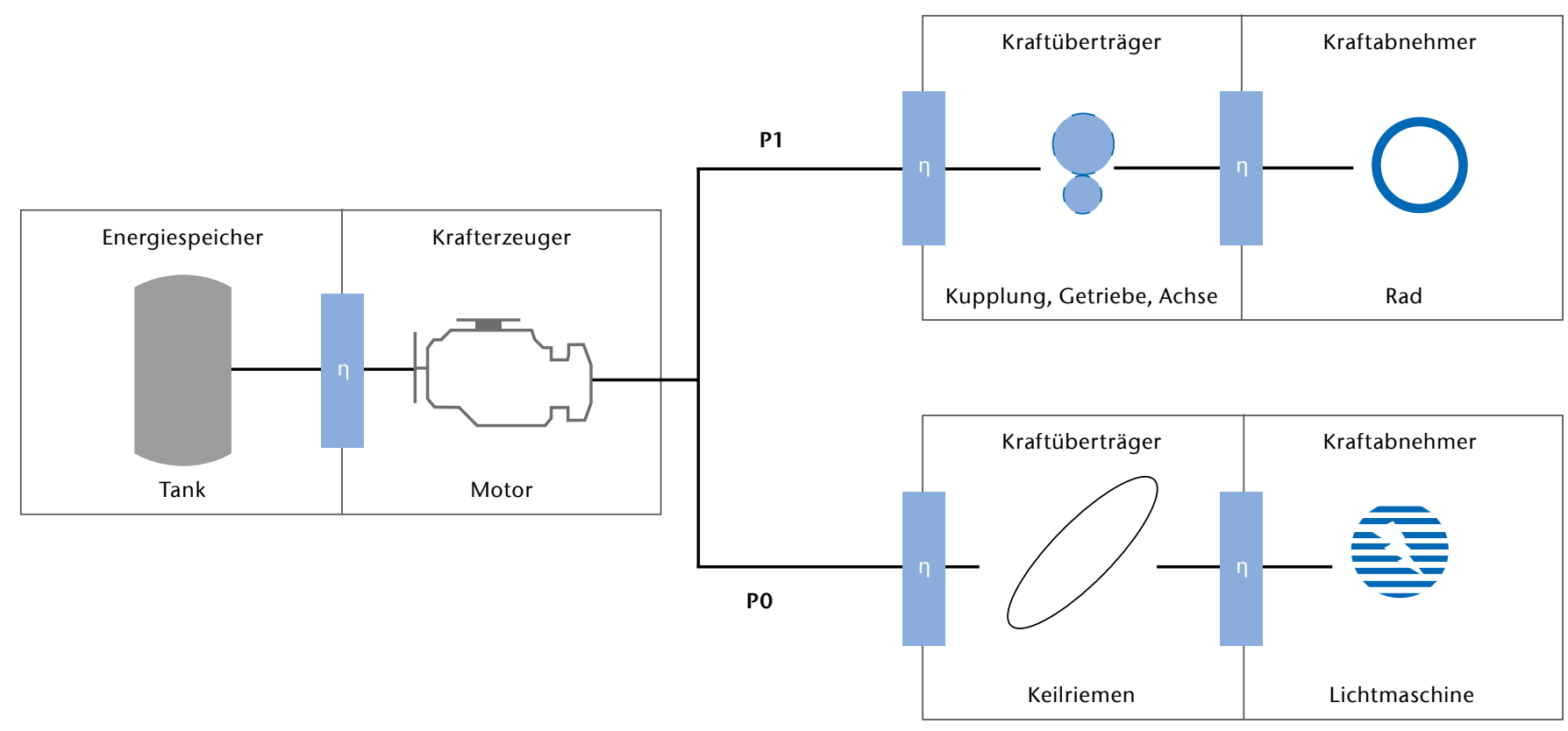

Abb. 1) Fahrzeugmodell, erweitert um Grundleistung und Wirkungsgrade.

sikalische Kräftemodell zurückgreift, gibt es doch eine Reihe von weiteren Publikationen, welche dieses um weitere offensichtlich relevante Aspekte erweitern. Davon ausgehend wurde die Entscheidung getroffen, noch einmal einen Schritt zurückzugehen und ausgehend von einer neutralen, rein physikalischen Betrachtung der Fahrzeugdynamik eine eigene Modellvariante zu entwickeln.

\section{MODELL ZUR ERMITTLUNG DES LEISTUNGSBEDARFS}

\subsection{VORBEREITUNG}

Die Beschreibung eines theoretischen Fahrzeugmodells, welches in einer zeitdiskreten mikroskopischen Verkehrssimulation eingesetzt werden kann, bedeutet, dass dieses Modell anhand von mathematischen und physikalischen Zusammenhängen aufgebaut wird und damit im Gegensatz zur experimentellen Modellbildung steht, in welcher die Modelle anhand der Identifikation von Ereignissen und Vorgängen erstellt werden. Der Schwerpunkt bei dieser Modellbildung liegt dabei auf einer möglichst korrekten Abbildung der physikalischen Zusammenhänge in Bezug auf das Fahrzeug. Somit werden in diesem Modell auch nur die technischen Faktoren berücksichtigt und nicht das Zusammenspiel zwi- schen mehreren Fahrzeugen oder psychologische Aspekte des Fahrzeugführers.

Als Ausgangspunkt dient ein einfaches allgemeines Fahrzeugmodell (siehe Abbildung 1), welches aus den vier Komponenten Energiespeicher, Krafterzeuger, Kraftübertragung und Kraftabnehmer besteht. Diese Verallgemeinerung soll es ermöglichen, die unterschiedlichsten Fahrzeugantriebsarten in der gleichen Simulationsumgebung abzubilden.

Der für den Vortrieb erforderliche Energieverbrauch eines Fahrzeugs, also die für den Kraftabnehmer erforderliche Energie zur Erfüllung der gestellten Aufgabe (z. B. gewünschte Geschwindigkeit), wird anhand eines physikalischen Kräftemodells bestimmt. Der äquivalente Leistungsbedarf, um das Fahrzeug anzutreiben, wird als $P_{1}$ bezeichnet.

Für eine realitätsnahe Modellierung ist es jedoch erforderlich, zusätzlich die elektrische Leistung zu berücksichtigten, welche für die im Fahrzeug verbauten Nebenverbraucher aufgewendet werden muss. Zu diesen Nebenverbrauchern gehören die elektrischen Verbraucher wie z.B. im Motor verbaute Pumpen, Servolenkung, Klimaanlage, Radio und Licht. Dieser Grundleistungsbedarf, also der von der Geschwindigkeit unabhängige Leistungsbedarf aller
Nebenverbraucher, wird als $P_{0}$ bezeichnet.

Bei der Betrachtung von Systemen aus der Perspektive des Leistungsbedarfs ist eine Einbeziehung von Wirkungsgraden ( $\eta$ ) pro Komponente oder Übertragungsweg unerlässlich, um die entstehenden Energieverluste durch Transformation oder Übertragung nicht zu vernachlässigen.

Der Aspekt der Laufzeitperformanz spiegelt sich zu diesem Zeitpunkt in der Form wider, dass im Rahmen des zu erstellenden Modells nur die in Längsrichtung auf das Fahrzeug einwirkenden Kräfte betrachtet werden.

\subsection{ALLGEMEINES PHYSIKALISCHES MODELL}

Das allgemeine physikalische Modell dient der Ermittlung des Leistungsbedarfs zum Antrieb der Räder, welcher durch die Antriebsmaschine eines Fahrzeugs für die Vorwärtsbewegung aufgewandt werden muss, um z. B. eine gewünschte Geschwindigkeit $v$ oder Beschleunigung a zu erreichen. Es handelt sich somit um die zu überwindende Bewegungskraft $F_{\text {Fahr }}$, welche auch als Fahrwiderstand bezeichnet wird. 
Nach Mitschke \& Wallentowitz (2004) wirken auf ein Fahrzeug in Fahrtrichtung insbesondere die folgenden Kräfte:

$F_{\text {Roll }} \quad$ als Rollwiderstand in $N$

$F_{\text {schwall }}$ als Schwallwiderstand in $N$

$F_{\text {Lager }} \quad$ als Lagerwiderstand in $N$

$F_{\text {RestBrems }}$ als Restbremsmoment in $N$

$F_{V s p} \quad$ als Vorspurwiderstand in $N$

$F_{\text {Kurve }}$ als Kurvenwiderstand in $N$

$F_{\text {steig }}$ als Steigungswiderstand in $N$

$F_{\text {Luft }}$ als Luftwiderstand in N

$F_{\text {Rot }} \quad$ als Rotationsenergie in N

$F_{\text {Besch }} \quad$ als Beschleunigungswiderstand in $N$

Für den Fahrwiderstand $F_{\text {Fahr }}$ gilt demnach

$$
\text { (1) } \begin{aligned}
F_{\text {Fahr }}= & F_{\text {Roll }}+F_{\text {Schwall }}+F_{\text {Lager }}+ \\
& F_{\text {RestBrems }}+F_{\text {Vsp }}+F_{\text {Kurve }}+F_{\text {Steig }}+ \\
& F_{\text {Luft }}+F_{\text {Rot }}+F_{\text {Besch }}
\end{aligned}
$$

Die nachfolgenden Abschnitte setzen sich mit den einzelnen Kräften auseinander und bewerten deren Relevanz.

\section{Rollwiderstand}

Der Rollwiderstand $F_{\text {Roll }}$ setzt sich aus zwei Komponenten zusammen. Zum einen aus der Verformungskraft, die der Reifen aufbringen muss, um sich in den Latsch hineinzudrehen bzw. wieder herauszudrehen. Diese Kraft entspricht der Radlast. Zum anderen aus der Reibekraft, die seitlich auf das Rad aufgrund der dauerhaften Verformung des Untergrundes auftrifft.

Da die Bestimmung der Reibekraft nur unter einem sehr hohen Aufwand erfolgen kann, wird zur Vereinfachung der Rollwiderstandsbeiwert als Proportionalitätsfaktor verwendet. Der Rollwiderstandsbeiwert wurde ursprünglich anhand von Versuchsergebnissen für verschiedene Rad-Untergrund-Kombinationen entwickelt und ist gängigen Tabellenbüchern zu entnehmen.

\section{Nach der Festlegung von}

$F_{\text {Rad }} \quad$ als die Radlast in $N$ und

$f_{r} \quad$ als den dimensionslosen Rollwiderstandsbeiwert lautet die Formel zur Bestimmung der Rollreibungskraft $F_{\text {Roll }}$ wie folgt:

$$
\text { (2) } F_{\text {Roll }}=F_{\text {Rad }} * f_{r}
$$

\section{Schwallwiderstand}

Der Schwallwiderstand tritt bei z.B. regennasser Fahrbahn auf und beschreibt die Kraft, die erforderlich ist, um Wasser unter dem Rad zu verdrängen. Die Größe des Widerstandes hängt von der Geschwindigkeit des fahrenden Fahrzeugs ab und von der Fläche, in die das Rad eintaucht. Die Bestimmung des Schwallwiderstandes erfolgt annäherungsweise.

\section{Nach der Festlegung von}

$v \quad$ als die Geschwindigkeit in $\mathrm{m} / \mathrm{s}$,

$b \quad$ als die Reifenbreite in $\mathrm{m}$ und

$n \quad$ als geschwindigkeitsabhängigen Exponenten

lautet die Formel zur Bestimmung des Schwallwiderstands $F_{\text {schwall }}$ wie folgt:

$$
\text { (3) } F_{\text {schwall }} \sim b^{*} v^{n}
$$

Der geschwindigkeitsabhängige Exponent kann gängigen Tabellenwerken entnommen werden und liegt laut Mitschke \& Wallentowitz (2004 : 12) ab einer Wasserstandshöhe von $0,5 \mathrm{~mm}$ bei ca. 1,6.

\section{Lagerreibung}

Der Lagerwiderstand $\left(F_{\text {Lager }}\right)$ tritt im Radlager auf und beschreibt eine Kraft, die entgegen der Rollrichtung wirkt. Zur Bestimmung ist das Wissen über die Lagerbelastung, den Reibbeiwert des Lagers und den Lagerradius erforderlich.

\section{Sei}

$F_{\text {LBelastung }}$ die Lagerbelastung in $N$, $\mu_{L} \quad$ der Reibbeiwert des Lagers und $r_{L} \quad$ der Lagerradius,

dann lautet die Formel für Bestimmung von $F_{\text {Lager }}$ in $\mathrm{Nm}$

$$
\text { (4) } F_{\text {Lager }}=\mu_{L} * r_{L} * F_{\text {Leelastung }}
$$

Obwohl in dieser Formel bereits Verallgemeinerungen getroffen wurden, wie z.B. der Einsatz eines Reibbeiwer- tes, über welchen das Material, die Schlüpfrigkeit und die Verarbeitungsqualität des Lagers abgebildet werden, kann diese Kraft nur unter einem sehr hohen Aufwand ermittelt werden. Abgesehen davon ist die Lagerreibung in der Regel, außer während des Anfahrens, sehr viel kleiner als die Rollreibung.

Um sie dennoch in einem Simulationsmodell einfließen lassen zu können, schlägt Neudorfer (2010:66) vor, einen Prozent der Radlast des Fahrzeugs als Näherungswert zu verwenden. Somit wäre

$$
\text { (5) } F_{\text {Lager }}=0,01 * F_{\text {Rad }}
$$

Exemplarische Berechnungen zeigen in diesem Fall jedoch einen deutlich zu hohen Anteil des Lagerwiderstands am Gesamtwiderstand, welcher als nicht korrekt eingeschätzt wird. In dieser Arbeit wird der Lagerwiderstand daher vernachlässigt.

\section{Restbremsmoment}

Mit dem Restbremsmoment $\left(F_{\text {RestBrems }}\right)$ wird die bei Scheibenbremsen auftretende Kraft beschrieben, die auch nach dem Lösen der Bremse auftreten kann. Dies geschieht in der Regel bei älteren Scheibenbremsen dadurch, dass die Bremsbeläge auf der Bremsscheibe schleifen (Mitschke \& Wallentowitz 2004).

Da das Restbremsmoment je nach Bauart und Alter der Bremsen signifikant variiert, wird dieser Wert in der Simulation vernachlässigt.

\section{Luftwiderstand}

Der Luftwiderstand beschreibt die Reibungskraft, die das Fahrzeug überwinden muss, um sich in der Erdatmosphäre bewegen zu können.

Nach der Festlegung von

$c_{w} \quad$ als dimensionslosen Strömungswiderstandskoeffizienten,

$\rho \quad$ als Luftdichte in $\mathrm{kg} / \mathrm{m}^{3}$,

$A \quad$ als Frontfläche des Fahrzeugs in $\mathrm{m}^{2}$ und

$v \quad$ als Geschwindigkeit in $\mathrm{m} / \mathrm{s}$ 
lautet die Formel zur Bestimmung des Luftwiderstandes $F_{\text {Luft }}$ folgendermaßen:

$$
6 F_{\text {Luft }}=1 / 2 * c_{w} * \rho * A * V^{2}
$$

Bei dieser Formel handelt es sich bereits um eine vereinfachte Betrachtung des Luftwiderstandes. So werden nur die bei absoluter Windstille frontal auf das Fahrzeug einwirkenden Kräfte betrachtet, die aufgrund der Luftverdrängung während der Fahrt auftreten.

Vernachlässigt werden einerseits Windgeschwindigkeiten und andererseits die seitlich und senkrecht auf das Fahrzeug einwirkenden Kräfte durch auftretende Winde und Luftverwirbelungen. Eine Berücksichtigung dieser Kräfte hätte Auswirkungen auf das gesamte Modell, da z.B. ein Aufwind unter dem Fahrzeug zu einer verringerten Radlast führen würde.

\section{Kurvenwiderstand}

Den Kurvenwiderstand ( $F_{\text {Kurve }}$ ) muss ein Fahrzeug während einer Kurvenfahrt überwinden. Während dieser bei normaler Fahrt unter $0,4 \mathrm{~g}$ auf trockener Straße als unbedeutend angesehen werden kann, sollten bei höheren Seitenbeschleunigungen die dadurch entstehenden Kräfte an den Antriebsrädern berücksichtigt werden.

\section{Nach der Festlegung von}

$m$ als Fahrzeugmasse in $\mathrm{kg}$,

$v \quad$ als Geschwindigkeit in $\mathrm{m} / \mathrm{s}$,

$r$ als Kreisradius in $m$,

$I, I_{H}, I_{V}$ als Abstand zwischen den beiden Achsen bzw. von vorderer oder hinterer Achse zum Fahrzeugschwerpunkt und

$a_{v}, \alpha_{H} \quad$ Schräglaufwinkel der Vorder- und Hinterräder

lautet die Formel zur Bestimmung des Kurvenwiderstands $F_{\text {Kurve }}$ wie folgt:

(7) $\begin{aligned} F_{\text {Kurve }}= & m * \frac{V^{2}}{r} *\left(\frac{I_{H}}{l} * \sin \left(a_{v}\right)+\right. \\ \left.\frac{I_{V}}{l} * \sin \left(\alpha_{H}\right)\right) & \end{aligned}$

Bei Annahme von $I_{H} / I=I_{V} / I$ vereinfacht sich dies $z u$

(8) $F_{\text {Kurve }}=m * \frac{v^{2}}{r} * \sin \left(\alpha_{v}\right)$
In der Arbeit von Hirano et al. (2014) werden die Kräfte einer Geradeausfahrt den Kräften einer Kurvenfahrt gegenübergestellt. Auf Abbildung 2 ist zu erkennen, dass sich die einwirkende Gesamtkraft während einer Kurvenfahrt (rechter Balken) im Gegensatz zur einwirkenden Kraft bei einer Geradeausfahrt (linker Balken) ungefähr verdoppelt.

Es lässt sich daraus schlussfolgern, dass der Kurvenwiderstand sehr wohl einen direkten Einfluss auf das Fahrzeugverhalten ausüben kann und somit zu einem erhöhten Leistungsbedarf führen kann.

\section{Steigungswiderstand}

Der Steigungswiderstand $\left(F_{\text {steig }}\right)$ beschreibt die positive Kraft, die dem Fahrzeug entgegenwirkt, wenn es eine Steigung befährt. Befährt das Fahrzeug ein Gefälle, wird diese Kraft negativ.

\section{Nach der Festlegung von}

$\begin{array}{ll}m & \text { als Fahrzeugmasse in } \mathrm{kg}, \\ g & \text { als Gravitationskraft in } \mathrm{m} / \mathrm{s}^{2} \text { und } \\ a & \text { als Steigungswinkel in } \mathrm{Grad}\end{array}$

lautet die Formel zur Bestimmung des Steigungswiderstandes $F_{\text {steig }}$ wie folgt:

(9) $F_{\text {steig }}=m * g * \sin (\alpha)$

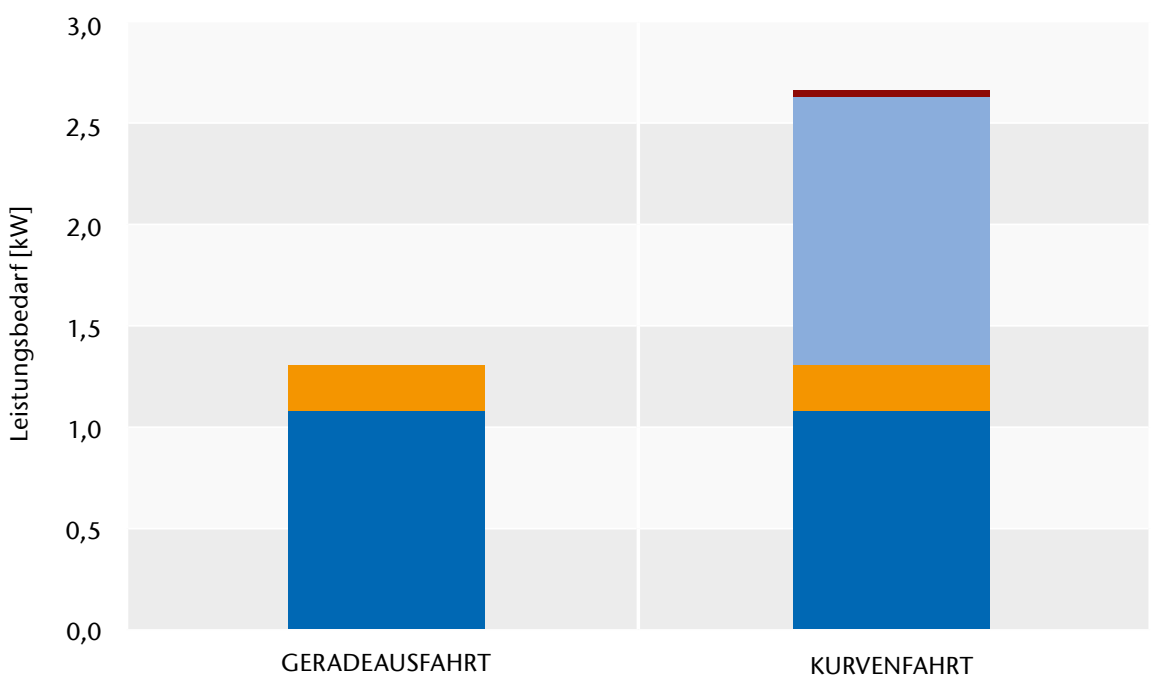

\section{Beschleunigungswiderstand der} Fahrzeugmasse

Die Beschleunigungskraft $\left(F_{\text {Beschl }}\right)$, auch Trägheitskraft genannt, tritt immer dann auf, wenn sich die Geschwindigkeit des Fahrzeugs verändert. Ganz konkret repräsentiert dieser Widerstand die translatorische Beschleunigung der Fahrzeugmasse.

\section{Nach der Festlegung von}

\section{$m \quad$ als Fahrzeugmasse in $\mathrm{kg}$ und \\ a als Beschleunigung in Fahrtrichtung in $\mathrm{m} / \mathrm{s}^{2}$}

lautet die Formel zur Bestimmung der Beschleunigungskraft $\left(F_{\text {Beschl }}\right)$ wie folgt:

$$
\text { (10) } F_{\text {Beschl }}=m * a
$$

\section{Beschleunigungswiderstand der rotatorischen Massen}

Der Beschleunigungswiderstand der rotatorischen Massen $\left(F_{\text {Rot }}\right)$ ist ein Teil des allgemeinen Beschleunigungswiderstands und beschreibt die erforderliche Kraft zur Beschleunigung der sich drehenden Teile eines Fahrzeugs. 
Nach der Festlegung von

$J_{R j} \quad$ als die Massenträgheitsmomente der sich drehenden Teile,

$r_{j} \quad$ als die Radien der sich drehenden Teile und

$R_{j} \quad$ als die dynamischen Halbmesser der sich drehenden Teile

lautet die Formel zur Bestimmung der Rotationsenergie $F_{\text {Rot }}$ wie folgt:

$11 F_{\text {Rot }}=a * \sum_{j=1}^{n} \frac{J_{R j}}{r_{j}{ }^{*} R_{j}}$

Es ist zu erkennen, dass die Berechnung der Rotationsenergie tiefgehendes Wissen über die einzelnen Komponenten des Fahrzeugs erfordert. Da der dafür zu betreibende Aufwand in keinem sinnvollen Verhältnis zum Nutzen steht, schlagen Maia et al. (2011) vor, diese Berechnung durch eine Anhebung der Fahrzeugmasse um $5 \%$ zu substituieren. Somit gilt:

(12) $F_{\text {Rot }}=0,05 * F_{\text {Besch }}$

\section{Zusammenfassung}

In der Betrachtung des Fahrwiderstands wurden die einzelnen Teilkräfte eingeführt und deren Berechnung beschrieben. Es wurde aufgezeigt, dass die Berechnung des Restbremsmoments, des Lagerwiderstandes und des Vorspurwiderstandes für die praktikable Bestimmung des Leistungsbedarfes einen so geringen Einfluss auf den gesamten Leistungsbedarf haben, dass sie vernachlässigt werden können. Außerdem wurde für die Bestimmung des Beschleunigungswiderstands der rotatorischen Massen eine Vereinfachung eingeführt. Darauf basierend kann Formel (1) folgendermaßen angepasst werden:

13 $\begin{aligned} F_{\text {Fahr }}= & F_{\text {Schwall }}+F_{\text {Roll }}+F_{\text {Kurve }}+F_{\text {Steig }}+ \\ & F_{\text {Luft }}+1,05 * F_{\text {Besch }}\end{aligned}$
3.3 BERÜCKSICHTIGUNG SIMULATIONSTECHNISCHER EINSCHRÄNKUNGEN

Moderne Verkehrssimulationen (vgl. Barceló 2010) sind sehr leistungsfähige Werkzeuge für eine Vielzah von Einsatzzwecken. Dessen ungeachtet gibt es immer noch eine Reihe von Beschränkungen für einzelne Einsatzfälle. Dies ist einer Reihe von Gründen geschuldet. So ist einerseits oftmals das vorhandene Kartenmaterial nicht aussagekräftig genug, um z.B. zuverlässige Höhenprofile $z u$ integrieren. Und andererseits erfordert - trotz aller Fortschritte - die vorhandene Rechenkapazität immer noch Kompromisse im Detailgrad der Modellierung von Straßennetzen.

Konkret hat dies die folgenden Auswirkungen auf das in (13) formulierte physikalische Modell:

- Die Verwendung von Graphen zur Modellierung des Straßennetzes bedingt die Zerlegung von Kurven in einzelne Teilstücke oder deren Beschränkung auf reine Knotenpunkte. Es sind daher in der Regel keine auf Kurvenradien basierende Funktionalitäten, wie z.B. das Abbremsen vor Kurven, realisierbar.

- Der Verzicht auf eine Integration von Wetterinformationen - höchstens auf globaler Ebene für das gesamte Simulationsgebiet - erlaubt daher auch nicht die realistische Integration von wetterbasierenden Krafteinflüssen. So sind z.B. Fahrbahnen mit stehendem Wasser üblicherweise nicht flächendeckend anzutreffen.

- Ohne realistische Höhenprofile fällt es schwer, zuverlässig die Einflüsse von Steigungen und Gefällen zu berücksichtigen. Um dieses Ziel zu erreichen müsste jedoch der Detailgrad des Knotennetzes derart erhöht werden, dass dies der Leistungsanforderung der Performanz widerspricht. Eine grobe Umsetzung ist jedoch möglich und wurde im Rahmen dieser Arbeit implementiert.
Unter Berücksichtigung der vorherigen Ausführungen ist es notwendig, der Modellierung des Fahrwiderstandes $F_{\text {Fahr }}$ folgende auch simulationstechnisch realisierbare Darstellung zu geben:

(14) $F_{\text {fahr }}=F_{\text {Roll }}+F_{\text {steig }}+F_{\text {Luft }}+1,05 * F_{\text {Besch }}$

Gleichwohl werden im nachfolgenden Vergleich die in (14) entfernten Einflüsse über pragmatische Annahmen und Nebenrechnungen berücksichtigt.

\section{VERGLEICH}

Für die Überprüfung der Relevanz unseres Modells haben wir es in einer mikroskopischen Verkehrssimulation implementiert und mit dem klassischen physikalischen Modell verglichen. Neben diesem Vergleich wurde die verwendete Simulationsumgebung derart modifiziert, um auch den entfallenen Schwallwiderstand sowie den Kurvenwiderstand zumindest vereinfacht berücksichtigen zu können.

\subsection{SIMULATIONSUMGEBUNG}

Die für die Durchführung der Simulation verwendeten statischen Fahrzeugmodellparameter sind in Tabelle 1 aufgeführt.

Die Fahrstrecke stellt einen dreieckigen Rundkurs dar. Die Eckpunkte der Strecke wurden A, B und C benannt. Ein Fahrzyklus besteht aus der Route $\mathrm{A} \rightarrow \mathrm{C} \rightarrow \mathrm{B} \rightarrow \mathrm{A} \rightarrow \mathrm{B} \rightarrow \mathrm{C} \rightarrow \mathrm{A}$. Der gewählte Fahrzyklus entspricht keinem Standardzyklus, wie z.B. dem Modifizierten Neuen Europäischen Fahrzyklus (MNEFZ) (vgl. Dietsche $\&$ Reif 2014), da diese Fahrzyklen in der Regel nur Bewegungen in verschiedenen Geschwindigkeiten auf einer Ebene beschreiben und somit die für diese Arbeit wesentlichen Auswirkungen von Steigungen und Gefällen außer Acht lassen. Detailinformationen über die Fahrstrecke enthält Tabelle 2 . 
Für die exemplarische Modellierung von Kurvenfahrten im Rahmen einer Verkehrssimulation wird ein stark abstrahiertes Kurvenmodell verwendet. Eine Kurve wird als Kreisbogen mit einem Radius $r$ und einem Mittelpunktswinkel $\alpha_{M}$ formuliert. Dies erlaubt dann die Berechnung der Länge des Kreisbogens und somit der für die Bewältigung dieser Kurve benötigten Leistung. Währenddessen wird ein konstanter Schräglaufwinkel $\alpha_{v}=5^{\circ}$ angenommen. Geschwindigkeitsänderungen auf den Hälften des Kreisbogens werden über die jeweils erlaubte Höchstgeschwindigkeit abstrahiert.

Die Festlegung der Kurvenradien in Tabelle 3 erfolgt gemäß den aktuelIen Baurichtlinien, veröffentlicht in Schneider et al. (2014).

\subsection{VERGLEICH DER LEISTUNGSBEDARFE}

Zum Vergleich der physikalischen Kräftemodelle wird ein Fahrzeug jeweils einen Fahrtzyklus lang auf der definierten Fahrtstrecke bewegt. Das Fahrzeugfolgemodell ist dabei derart ausgelegt, dass es immer versucht, die jeweilige erlaubte Höchstgeschwindigkeit zu erreichen, jedoch Bremswege vor Geschwindigkeitseinschränkungen oder dem Ziel berücksichtigt. Während der Fahrt werden die Leistungswerte für die Überwindung der jeweiligen Widerstände für jeden Simulationsschritt $(\Delta t=20 \mathrm{~ms})$ erfasst.

\section{Verglichen werden die folgenden Kräftemodelle:}

a) das klassische Modell von Treiber \& Kesting (2013)

b) das in dieser Arbeit entwickelte Modell (siehe (14))

c) Formel (14), erweitert um einen konstanten Schwallwiderstand (mit $\mathrm{n}=1,5$ und $\mathrm{b}=0,195 \mathrm{~m}$ )

d) Formel (14) mit Berücksichtigung des Kurvenwiderstands

Zuerst stellt der für eine Fahrt benötigte Gesamtleistungsbedarf die Grundlage für den Vergleich dar. Die gewonnenen Ergebnisse zeigen, dass die in dieser Arbeit erstellten Modelle insgesamt gesehen keinen signifikanten Unterschied bei der Modellierung
Tab. 1: Statische Modellparameter

\begin{tabular}{|l|c|c|}
\hline \multicolumn{1}{|c|}{ Parameter } & Symbol & Wert \\
\hline Fahrzeuggewicht & $m$ & $1400 \mathrm{~kg}$ \\
\hline Maximale Geschwindigkeit & $v_{\text {max }}$ & $192 \mathrm{~km} / \mathrm{h}$ \\
\hline Maximale Beschleunigung & $a_{+\max }$ & $2 \mathrm{~m} / \mathrm{s}^{2}$ \\
\hline Maximale Bremsrate & $a_{-\max }$ & $3 \mathrm{~m} / \mathrm{s}^{2}$ \\
\hline Strömungswiderstandskoeffizient & $c_{w}$ & 0,3 \\
\hline Fahrzeugfrontfläche & $A$ & $2,0 \mathrm{~m}$ \\
\hline Rollwiderstandsbeiwert & $f_{r}$ & 0,01 \\
\hline Luftdichte bei $20^{\circ}$ & $\rho$ & $1,25 \mathrm{~kg} / \mathrm{m}^{3}$ \\
\hline
\end{tabular}

Tab. 2: Statische Modellparameter

\begin{tabular}{|c|c|c|c|}
\hline Strecke & Länge & Steigung & Max. Geschwindigkeit \\
\hline A - B & $14.993 \mathrm{~m}$ & $6,48 \%$ & $30 \mathrm{~km} / \mathrm{h}$ \\
\hline B - C & $16.054 \mathrm{~m}$ & $0,00 \%$ & $80 \mathrm{~km} / \mathrm{h}$ \\
\hline A - C & $29.994 m$ & $3,24 \%$ & $50 \mathrm{~km} / \mathrm{h}$ \\
\hline
\end{tabular}

Tab. 3: Kurvendefinition

\begin{tabular}{|c|c|c|}
\hline Kurve & Mittelpunktswinkel & Radius \\
\hline A - B - C & $150,05^{\circ}$ & $200 \mathrm{~m}$ \\
\hline B - C - A & $14,45^{\circ}$ & $300 \mathrm{~m}$ \\
\hline B - A - C & $15,50^{\circ}$ & $90 \mathrm{~m}$ \\
\hline
\end{tabular}

Tab. 4: Vergleich der Gesamtleistungsbedarfe

\begin{tabular}{|l|l|l|l|l|}
\hline & Modell (a) & Modell (b) & Modell (c) & Modell (d) \\
\hline \begin{tabular}{l|l|l|l|}
\hline Beschleunigungswiderstand \\
Rollwiderstand
\end{tabular} & $63776 \mathrm{~kW}$ & $64178 \mathrm{~kW}$ & $64966 \mathrm{~kW}$ & $64178 \mathrm{~kW}$ \\
\hline $\begin{array}{l}\text { Steigungswiderstand } \\
\text { Luftwiderstand }\end{array}$ & $619173 \mathrm{~kW}$ & $418835 \mathrm{~kW}$ & $418834 \mathrm{~kW}$ & $418835 \mathrm{~kW}$ \\
\hline $\begin{array}{l}\text { Beschleunigungswiderstand } \\
\text { (rot.) }\end{array}$ & $351055 \mathrm{~kW}$ & $351004 \mathrm{~kW}$ & $350999 \mathrm{~kW}$ & $351004 \mathrm{~kW}$ \\
\hline $\begin{array}{l}\text { Schwallwiderstand } \\
\text { Kuvenwiderstand }\end{array}$ & $3209 \mathrm{~kW}$ & $3248 \mathrm{~kW}$ & $3209 \mathrm{~kW}$ \\
\hline & & & $205 \mathrm{~kW}$ & \\
\hline
\end{tabular}


der verschiedenen Kräfteeinflüsse erzielen (siehe Tabelle 4).

Im Einzelnen zeigt sich zum Beispiel bei der Betrachtung des Rollwiderstandes, dass die von Treiber \& Kesting getroffenen Vereinfachungen, d.h. die Nichtberücksichtigung des Steigungswinkels zur Anpassung der Normalkraft, wirklich gerechtfertigt sind. Der Anteil des Beschleunigungswiderstandes der rotatorischen Massen beträgt erwartungsgemäß 5,0 Prozent. Eine weitere Ursache für die geringfügigen Unterschiede ist in den stochastischen Elementen des Fahrzeugfolgemodells (z.B. Trödelfaktor) zu suchen.

Ein ganz anderes Bild ergibt sich jedoch bei der Betrachtung einer situativen Energiebilanz. Wird - dies sei an dieser Stelle exemplarisch herausgegriffen - der Energiebedarf einer einzelnen Zeitscheibe verglichen, so wird deutlich, dass Kräfte wie der Kurvenwiderstand situativ eine signifikante Rolle spielen können. Ein im Modell (d) im Gegensatz zu Modell (a) um 27,1 Prozent erhöhter momentaner Leistungsbedarf bei einer Kurvenfahrt in der Ebene mit konstanter Geschwindigkeit (siehe Abbildung 3) kann zum Beispiel dazu führen, dass - auch bei klassischen Fahrzeugkonzepten - die Maximalleistung des Antriebs überschritten wird und die aktuelle Ge- schwindigkeit nicht gehalten werden kann. Fahrzeuge mit kombinierten Antrieben könnten u.a. mit einer Veränderung des Betriebsmodus, d.h. z.B. Zuschaltung eines weiteren Antriebs, reagieren.

\section{ABSCHLUSSBETRACHTUNGEN}

Bei dem Vergleich der Ergebnisse der verschiedenen Modellierungsvarianten konnte festgestellt werden, dass die primär sowohl aus dem Grund der Vereinfachung des Modells als auch der Berechnung im Allgemeinen nicht berücksichtigten Komponenten des physikalischen Modells keinen signifikanten Einfluss auf den Gesamtenergiebedarf für die Bewegung von Fahrzeugen haben. Für den Energiebedarf im Momentanbereich konnte jedoch eine Relevanz deutlich aufgezeigt werden.

Die üblicherweise getroffenen Vereinfachungen sind aus Sicht der Zielstellung vieler Simulationsprojekte verständlich. Wenn ein allgemeines Emissionsmodell für Fahrzeuge mit Verbrennungsmotor aufgestellt oder die großflächige Verteilung von Ladestationen bei Elektrofahrzeugen untersucht werden soll, dann sind derlei Vereinfachungen sehr wohl vertretbar, da ihre Signifikanz durch den gewünschten bzw. geforderten Genauigkeitsgrad aufgehoben wird.
Bei der Simulation von Fahrzeugen mit alternativen Antrieben ist es jedoch erforderlich, deren interne Prozesse des Energiemanagements möglichst genau abzubilden. Die Steuerung des Zusammenspiels von mehreren Antrieben ist ein komplexes Geflecht von Regeln und Zuständen, dessen Modellierung wesentlich stärker von externen und internen situativen Parametern, dabei gerade auch vom aktuellen Leistungsbedarf, abhängt, als dies bei Fahrzeugen mit nur einem Antrieb erforderlich ist. So führt etwa die in dem erstellten Modell erzielte Erhöhung des Anteils der Energien welche nicht rekuperiert werden können, d.h. Energien, welche das System Fahrzeug verlassen, auch dazu, dass z.B. „Folgefehler“ bei der Ermittlung der Reichweite vermieden bzw. verringert werden.

Zusammenfassend gesehen muss daher konstatiert werden, dass für eine weitere Verbesserung der Simulationsqualität, gerade im Hinblick auf Fahrzeuge mit alternativen Antrieben, an einer Reduzierung der funktionstechnischen Einschränkungen von Verkehrssimulationsumgebungen gearbeitet werden muss. Lässt sich dieser Schritt nicht vollziehen, dann sind die Vereinfachungen des klassischen Energiebedarfsmodells (siehe Modell (a)) als geeignet anzusehen.

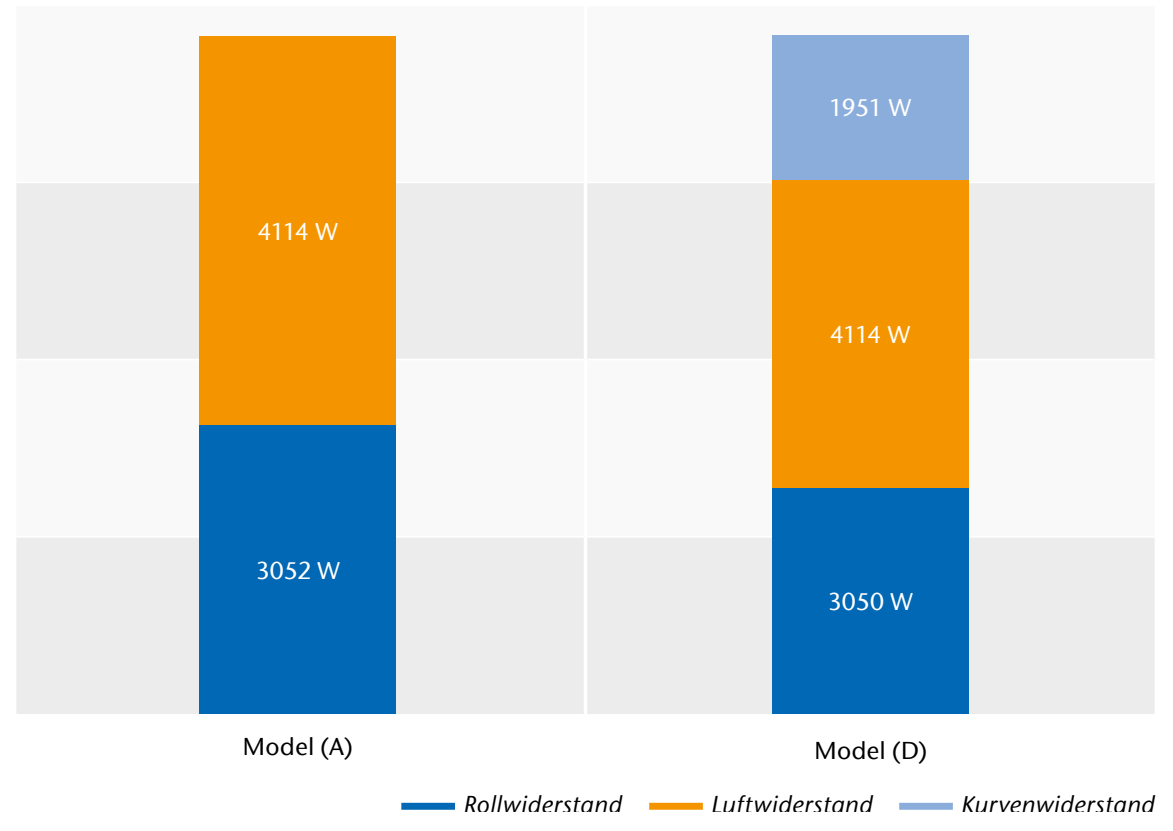

Abb. 3) Situativer Leistungsbedarf bei konstanter Kurvenfahrt in der Ebene.

${ }^{2}$ Die Zeitscheiben ergeben sich aufgrund der diskreten Simulationsarchitektur. 


\section{LITERATUR}

Barceló J (ed) (2010) Fundamentals of Traffic Simulation. International Series in Operations Research \& Management Science, vol 145. Springer, New York. ISBN: 978-14419-6142-6. doi: 10.1007/978-1-4419-6142-6

Die Bundesregierung (2009) Nationaler Entwicklungsplan Elektromobilität der Bundesregierung. http:// www.bmbf.de/pubRD/nationaler_entwicklungsplan elektromobilitaet.pdf. Accessed 17 Sep 2014

Dietsche K, Reif K (2014) Kraftfahrtechnisches Taschenbuch, 28th edn. Springer Vieweg, Wiesbaden. ISBN: 978-3-658-03800-7

Hirano Y, Inoue S, Ota J (2014) Model-based Development of Future Small EVs using Modelica. In Tummescheit H, Årzén K (eds) Proceedings of the 10th International Modelica Conference, 10-12 March 2014, Lund, Sweden, pp 63-70. doi: 10.3384/ecp1409663

Kraftfahrt-Bundesamt (2014a) Der Fahrzeugbestand im Überblick am 1. Januar 2014 gegenüber 1. Januar 2013. http://www.kba.de/DE/Statistik/ Fahrzeuge/Bestand/2014_b_ueberblick_pdf.pdf? blob=publicationFile\&v=7. Accessed 17 Sep 2014

Kraftfahrt-Bundesamt (2014b) Jahresbilanz der Neuzulassungen Jahr 2013. http://www.kba.de/DE/Statistik/ Fahrzeuge/Neuzulassungen/neuzulassungen_inhalt. html?nn=644264. Accessed 17 Sep 2014

Kurczveil T, Schnieder E (2013) Extending a traffic simulation tool for the evaluation of novel charging infrastructures. In: 3rd International Electric Drives Production Conference (EDPC), 29-30 Oct 2013, Nuremberg, Germany, pp 1-5. doi: 10.1109/EDPC.2013.6689752

Maia R, Silva M, Araujo R, Nunes U (2011) Electric vehicle simulator for energy consumption studies in electric mobility systems. In: IEEE Forum on Integrated and Sustainable Transportation Systems (FISTS), 29 Jun - 1 Jul 2011, Vienna, Austria, pp 227-232. doi: 10.1109/ FISTS.2011.5973655

Mitschke M, Wallentowitz H (2004) Dynamik der Kraftfahrzeuge, 4 th edn. Springer, Berlin. ISBN: 978-3540-42011-8

Neudorfer H (2010) Weiterentwicklung von elektrischen Antriebssystemen für Elektro- und Hybridstraßenfah zeuge. Österreichischer Verband für Elektrotechnik, Wien. ISBN: 978-3-85133-056-4

Schellenberg S, Berndt R, Eckhoff D, German R (2014) A Computationally Inexpensive Battery Model for the Microscopic Simulation of Electric Vehicles. In: IEEE 80th Vehicular Technology Conference (VTC Fall), 14-17 Sep 2014, Vancouver, BC, Canada, pp 1-6. doi: 10.1109/ VTCFall.2014.6965807

Schneider K, Goris A, Albert A (eds) (2014) Bautabellen für Ingenieure. Mit Berechnungshinweisen und Beispielen, 21st edn. Bundesanzeiger-Verlag, Köln. ISBN 978-3-8462-0304-0

Toyota Deutschland GmbH (2014) Prius - Preise und Fakten. http://www.toyota.de/download/cms/dede/ Toyota-Prius-PDB_tcm-17-100397.pdf. Accessed $17 \mathrm{Sep}$ 2014

Treiber M, Kesting A (2010) Verkehrsdynamik und -simulation. Daten, Modelle und Anwendungen der Verkehrsflussdynamik. Springer, Berlin, Heidelberg. ISBN: 978-3-642-05227-9. doi: 10.1007/978-3-642-05228-6

Treiber M, Kesting A (2013) Traffic flow dynamics. Data, models and simulation. Springer, Heidelberg, New York. ISBN: 978-3-642-32460-4

\section{AUTOREN}

Peter Bernhardt, M.Sc

Technologietransfer- und Weiterbildungszentrum an

der Technischen Hochschule Wildau FH e.V.

peter.bernhardt@th-wildau.de

\section{Christian Sauer, M.Sc.}

Technologietransfer- und Weiterbildungszentrum an

der Technischen Hochschule Wildau FH e.V.

sauer@th-wildau.de

\section{Prof. Dr. Michael Hendrix}

Technische Hochschule Wildau

michael.hendrix@th-wildau.de 